\title{
Diverse functions of uterine proteoglycans in human reproduction (Review)
}

\author{
KOTARO KITAYA $^{1,2}$, YOSHIHIRO TADA ${ }^{1}$, TERUMI HAYASHI ${ }^{1}$, SAGIRI TAGUCHI $^{1}$, \\ MIYAKO FUNABIKI $^{1}$, YOSHITAKA NAKAMURA ${ }^{1}$ and TADAHIRO YASUO ${ }^{3}$ \\ ${ }^{1}$ IVF Center, Oak Clinic, Osaka 557-0045; ${ }^{2}$ Department of Anatomy and Cell Science, Kansai Medical University, \\ Osaka 570-8506; ${ }^{3}$ Department of Obstetrics and Gynecology, Graduate School of Medical Science, \\ Kyoto Prefectural University of Medicine, Kyoto 602-8566, Japan
}

Received December 27, 2011; Accepted March 1, 2012

DOI: $10.3892 / \mathrm{mmr} .2012 .826$

\begin{abstract}
Proteoglycans (PGs) are a group of heavily glycosylated proteins that are present throughout the mammalian body and are involved in a wide variety of biological phenomena, including structural maintenance, tissue remodeling, molecular presentation, cell adhesion and signal transmission. Previous studies have revealed an increasing number of roles for PGs in human reproduction. Several PGs are currently utilized or regarded as biomarkers for the diagnosis of certain pathological uterine conditions associated with infertility and obstetrical complications. The aim of this review was to discuss the involvement of PGs in the human uterus in reproductive biology and pathophysiology.
\end{abstract}

\section{Contents}

1. Introduction

2. PGs in human endometrium

3. Close association between miscarriage and deficiency in thrombomodulin and syndecan-4

4. Syndecan-1 as a diagnostic biomarker for plasmacyte infiltrates in chronic endometritis

5. DSPGs mediate ectopic growth and development of endometriosis

6. PGs in human myometrium and cervix

7. Contribution of fibromodulin, versican and decorin to structural maintenance and growth of uterine fibroids

8. Low myometrial syndecan-3 expression in dystocia

9. PGs in human placenta

10. Amniotic fluid perlecan and agrin as potential biomarkers for premature rupture of fetal membranes

Correspondence to: Dr Kotaro Kitaya, IVF Center, Oak Clinic, 2-7-9 Tamade-nishi, Nishinari-ku, Osaka 557-0045, Japan

E-mail: kitaya@koto.kpu-m.ac.jp

Key words: glycosaminoglycan, proteoglycan, reproduction, uterus
11. Preventive role of syndecan-1 against pre-eclampsia

12. Upregulation of perlecan in the villi of gestational diabetes mellitus

13. Elevated amniotic fluid lumican concentration in fetus with Turner syndrome

14. Conclusions

\section{Introduction}

Proteoglycans (PGs) are a group of macromolecules which consist of one core protein and one or more covalently attached sulfated glycosaminoglycan (GAG) side chain(s). The PG core proteins are encoded by distinct genes and contain multiple functional domains that bind to and modulate a wide range of molecules, including chemokines, cytokines, growth factors, extracellular matrix (ECM) proteins, coagulation factors and lipid-metabolizing enzymes. The localization of PGs is attributed to the molecular and structural characteristics of their core proteins. GAGs consist of diversely epimerized/sulfated unbranched polysaccharide chains composed of 1,4-linked repeated disaccharide units. According to the combination of disaccharides, the sulfated GAG side chains are classified as chondroitin sulfate (CS), dermatan sulfate (DS), heparan sulfate (HS) and keratan sulfate (KS). The sulfation status of the GAG side chains in PGs varies with tissue type, location and age, which provides PGs with organ-dependent properties in molecular weight, structure and function (1).

PGs are classified into two distinct types by their location in tissues: i) cell surface PGs and ii) ECM PGs (Table I). The cell surface PGs include the syndecan and glypican families. Syndecans are a group of transmembrane HSPGs that interact with and regulate various soluble molecules. Glypicans are glycosylphosphatidylinositol-anchored HSPGs on the outer surface of the plasma membrane that regulate multiple intracellular signal pathways. The ECM PGs are subdivided into small leucine-rich PGs, the aggrecan/versican family and basement membrane PGs. Small leucine-rich PGs, which share repeats of leucine-rich structural motifs and cysteine residues, contribute to collagen fibrillogenesis and matrix assembly in the ECM. The aggrecan/versican family is a group of large PGs that engage in the maintenance of osmotic pressure and 
the transpresentation of soluble molecules to other cells, while basement membrane PGs are involved in cell structure and angiogenesis (1).

PGs are broadly distributed in mammalian tissues. Previous studies have revealed the critical involvement of PGs in embryogenesis and organogenesis, particularly of cartilage, bone and eyes. Since the female genital organs exhibit marked physiological turnover and remodeling under the influence of ovarian steroids and pituitary hormones, PGs are thought to affect multiple facets of reproduction. Despite emerging data concerning the expression and functions of PGs in the genital organs in animal models, the exact roles of PGs in human reproduction have yet to be determined. The aim of this review was to discuss the current understanding of the correlation between PGs in the human uterus and reproduction and to provide insights into the possible future clinical uses of these studies.

\section{PGs in human endometrium}

Early studies focused on the expression of HSPGs in the endometrium and their effects on embryo implantation. In the human endometrium, HS is localized mainly to the basement membranes of the glandular epithelium, microvascular endothelium and stromal compartment and the ECM of the decidualized endometrium. HS expression in the endometrial glandular epithelium is constant throughout the menstrual cycle, while that in the endometrial microvascular endothelium acutely decreases during menstruation $(2,3)$. By contrast, $\mathrm{KS}$ is absent in the proliferative phase endometrium, but appears in the surface/glandular epithelium and gland secretion during the secretory phase (4). DS is localized to the whole endometrium and its expression level in microvascular endothelial cells and stromal fibroblasts increases following ovulation (5). Previous studies have reported that CS is the predominant sulfated GAG species in the human endometrium. CS is localized to the stromal compartment of the cycling endometrium, particularly around arterioles and glands, with no marked menstrual cycledependent fluctuation (6).

The production of certain PGs in the human endometrium is regulated by ovarian steroids. For instance, the HSPG thrombomodulin is among the genes induced in endometrial explant culture by in vitro progesterone stimulation (7). It has been demonstrated that progesterone (alone or in combination with $17 \beta$-estradiol) enhances the expression of CS/HSPG serglycin and DS/CSPG biglycan and versican in endometrial endothelial cells (8). Another potential regulator of endometrial PG synthesis is TGF- $\beta 3$, which is capable of suppressing HSPG expression in endometrial stromal fibroblasts (9).

As yet, no study has proven the indispensability of the endometrial PGs in embryo implantation. Mice lacking one of the PG core proteins or GAG-biosynthetic enzymes have been generated, but these mice develop normal primal embryo attachment sites on the decidualizing endometrium. As glypican-1 is a functional ligand for the prostaglandin-synthetic enzyme secretory phospholipase A2 group IIA, which is crucial for embryo attachment, endometrial glypican-1 was suggested as a candidate PG responsible for embryo implantation. The endometrial expression level of glypican-1, however, was similar in fertile women and patients with repeated implantation failure following in vitro fertilization-embryo transfer (10). These findings are likely to result from the overlapping properties of PGs in embryo implantation.

By contrast, certain HSPGs and HS-synthetic enzymes are critical in early placentation. These topics are discussed below. Of note, the CS/DSPGs versican, biglycan and serglycin also potentially contribute to early placentation. Their expression level in the endometrial endothelium increases following ovulation $(5,11)$. These endometrial endothelial PGs are thought to initiate the selective recruitment of circulating CD16(-) natural killer cells, which uniquely accumulate in the endometrial stromal compartment following ovulation (12). These locally extravasated natural killer cells have been demonstrated to be essential for early placentation, as they secrete a wide variety of chemokines and angiogenic factors as well as monitoring trophoblast invasion (13).

\section{Close association between miscarriage and deficiency in thrombomodulin and syndecan-4}

Thrombomodulin was first cloned as a coagulation cascade co-factor expressed by intact endothelial cells. The membrane-bound form of thrombomodulin binds thrombin, which in turn catalyzes protein $\mathrm{C}$ and thrombin activatable fibrinolysis inhibitor into their mature forms and induces anti-inflammatory responses and cytoprotective signals in endothelial cells. In the murine uterus, thrombomodulin is expressed in the developing vasculature of embryos as well as in giant trophoblasts. Despite intact organogenesis and vasculogenesis, mice deficient in thrombomodulin exhibit embryonic lethality during mid to late gestation. These embryos exhibit consumptive coagulopathy during this period when massive hemorrhage occurs in the uterus, indicating the critical engagement of thrombomodulin in anticoagulant activity in the placenta (14). In humans, the presence of thrombomodulin has been confirmed in decidual endothelial cells in the first trimester of pregnancy (15). The results in the thrombomodulin gene knockout mice were confirmed by a prospective case-control study which demonstrated that the gene transcript expression level of thrombomodulin is lower in the placenta of unexplained recurrent miscarriage compared with that of normal pregnancy (16).

Moreover, mice lacking the HSPG syndecan-4 gene are prone to form thrombi at the fetal-maternal interface, leading to miscarriage. The placental bed thrombosis in these mice is caused by the impaired bioactivity of anticoagulant factor anti-thrombin III, which is bound to and regulated by syndecan-4 (17). Syndecan-4 thus contributes to the maintenance of pregnancy by exerting coagulofibrinolytic activity disparate from thrombomodulin. In humans, syndecan-4 is localized to glandular epithelial cells in the cycling endometrium (18), but its involvement in placentation remains to be established.

\section{Syndecan-1 as a diagnostic biomarker for plasmacyte infiltrates in chronic endometritis}

Syndecan-1 is involved in the presentation and modulation of ECM proteins (collagen types I, III and V, fibronectin and integrins), anticoagulation factors (thrombospondin and 
Table I. Expression profiling of PGs in the human non-pathological uterus. ${ }^{\text {a }}$

\begin{tabular}{|c|c|c|c|c|c|c|}
\hline & \multicolumn{3}{|c|}{ Non-pregnant condition } & \multicolumn{3}{|c|}{ Pregnant condition } \\
\hline & Endometrium & Myometrium & Cervix & Decidua & Myometrium & Placenta \\
\hline \multicolumn{7}{|l|}{ Cell surface PGs } \\
\hline \multicolumn{7}{|l|}{ Syndecan family } \\
\hline 1 & + & - & + & - & + & + \\
\hline 2 & + & + & NA & + & + & + \\
\hline 3 & + & + & NA & NA & + & - \\
\hline 4 & + & + & NA & + & + & + \\
\hline \multicolumn{7}{|l|}{ Glypican family } \\
\hline 1 & + & + & + & + & + & + \\
\hline 3 & NA & - & - & $+^{\mathrm{b}}$ & NA & + \\
\hline \multicolumn{7}{|l|}{ Others } \\
\hline TGF- $\beta$ receptor III & + & $+/ \mathrm{NA}$ & $+/ \mathrm{NA}$ & + & $+/ \mathrm{NA}$ & $+/ \mathrm{NA}$ \\
\hline CSPG5 (neuroglycan) & - & NA & NA & NA & NA & NA \\
\hline \multicolumn{7}{|l|}{ ECM PGs } \\
\hline \multicolumn{7}{|c|}{ Small leucine-rich PG family } \\
\hline \multicolumn{7}{|l|}{ Class I } \\
\hline Biglycan & + & + & + & NA & + & + \\
\hline Decorin & + & + & + & NA & + & + \\
\hline ECM2 & + & + & NA & NA & NA & NA \\
\hline Asporin & $\mathrm{NA}^{\mathrm{c}}$ & $\mathrm{NA}^{\mathrm{c}}$ & $\mathrm{NA}^{\mathrm{c}}$ & NA & NA & NA \\
\hline \multicolumn{7}{|l|}{ Class II } \\
\hline Fibromodulin & NA & + & NA & NA & NA & NA \\
\hline Lumican & NA & NA & + & NA & NA & + \\
\hline \multicolumn{7}{|l|}{ Class III } \\
\hline Osteoglycin & NA & NA & NA & + & NA & NA \\
\hline Epiphycan & - & NA & NA & NA & NA & NA \\
\hline \multicolumn{7}{|l|}{ Class IV } \\
\hline Nyctalopin & - & - & - & NA & NA & - \\
\hline \multicolumn{7}{|l|}{ Others } \\
\hline Tsukushi & NA & NA & NA & NA & NA & NA \\
\hline \multicolumn{7}{|l|}{ Aggrecan/versican family } \\
\hline Aggrecan & + & NA & + & NA & NA & NA \\
\hline Versican & + & + & + & NA & NA & + \\
\hline Neurocan & - & NA & NA & NA & NA & NA \\
\hline Brevican & - & NA & NA & NA & NA & NA \\
\hline \multicolumn{7}{|l|}{ Basement membrane PGs } \\
\hline HSPG2 & + & + & + & + & + & + \\
\hline Agrin & NA & NA & NA & + & NA & + \\
\hline Collagen type XVIII A1 & NA & NA & NA & NA & NA & - \\
\hline \multicolumn{7}{|l|}{ Others } \\
\hline Serglycin & + & NA & NA & NA & NA & NA \\
\hline Testican-1 & NA & + & NA & NA & NA & NA \\
\hline
\end{tabular}

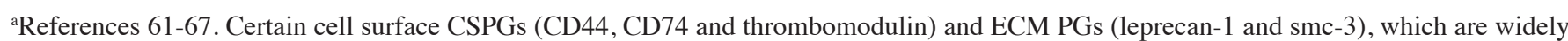
expressed in various somatic cell types, are not listed here. The expression of the gene transcripts for several small leucine-rich PGs (keratocan, osteomodulin, prelp, opticin and chondroadherin) and basement membrane PGs testican-2 and -3 and collagen family members (types IX, XII, $\mathrm{XIV}$ and XV) are not detectable. ${ }^{b}$ Limited to the pseudodecidualized endometrium in ectopic pregnancy. ${ }^{c}$ Confirmed at the gene transcript level, but not at the protein level. PG, proteoglycan; CSPG, chondroitin sulfate PG; ECM, extracellular matrix. NA, not applicable. 
anti-thrombin III) and vascular endothelial growth factors. In the human non-pregnant uterus, syndecan-1 is localized to the cervix and endometrium, but not to the myometrium (18-22). In the human endometrium, the expression level of syndecan-1 in endometrial epithelial cells is higher in the secretory phase than in the proliferative phase, while that in endometrial stromal fibroblasts is constant throughout the menstrual cycle. The localization patterns of syndecan-1 in the human endometrium are inconsistent between studies; some authors suggest that it is confined mainly to the apical and basolateral surface in glandular epithelial cells only during the secretory phase, while other authors claim that it has a wide distribution including surface/glandular epithelial cells and stromal fibroblasts (18,20-22). Such discrepancies appear to result from the differences in the antibodies used (i.e., mouse monoclonal antibody versus goat polyclonal antibody) and tissue preparation methods (i.e., frozen tissue versus fixed tissue).

Syndecan-1 has been established as a specific and sensitive biomarker for endometrial stromal plasmacyte infiltrates and is the most reliable histopathological landmark for chronic endometritis (18). Immunohistochemistry for syndecan-1 improved the diagnostic accuracy of chronic endometritis, a potential cause of repeated embryo implantation failure (following in vitro fertilization and embryo transfer) and recurrent miscarriage (23-25). In multiple myeloma, syndecan-1 inhibits tumor cell invasion by suppressing the bioactivity of matrix metalloproteinase-9, which is a protease that is highly expressed in the endometrium of infertile women (26). It has been shown that syndecan-1-positive endometrial plasmacytes, unlike certain aggressive syndecan-1-negative endometrial B cells, remain in the stromal compartment without invading and destroying epithelial basement membranes (24). These findings suggest the protective role of syndecan-1 in the maintenance of endometrial integrity against viral infections including human immunodeficiency virus-1, which initially attaches to endometrial epithelial cells via the cell surface HSPGs and frequently causes chronic endometritis (27).

\section{DSPGs mediate ectopic growth and development of endometriosis}

Endometriosis involves the ectopic growth and development of endometrial component cells. Ectopic endometrial tissues often deteriorate female fecundity by spreading over entire reproductive organs, ranging from the myometrium, peritoneum and ovaries to the Fallopian tubes. In contrast to eutopic endometrium being predominated by CS, DS is the major sulfated GAG observed in deeply infiltrating endometriotic lesions (28).

DSPG versican is a gene that is highly expressed in the eutopic endometrium of endometriosis. In addition, the expression level of versican in the eutopic endometrial epithelium during the midsecretory phase is higher in severe cases of endometriosis than in mild cases $(29,30)$. As versican is capable of stimulating cell proliferation and inhibiting apoptosis by binding ECM proteins on the cell surface, versican may contribute to the survival and increase in the number of eutopic endometrial cells in the ectopic microenvironment.

DS/CS-bearing PG CD44 forms a complex with another CSPG, CD74, and triggers the mitogen-activated protein kinase signaling cascades for cell survival. In endometriotic cells, macrophage migration-inhibitory factor has been demonstrated to bind the CD44/CD74 complex and activate chemokines and angiogenic factors in these cells, suggesting the involvement of this PG complex in the growth and development of endometriotic lesions (31).

\section{PGs in human myometrium and cervix}

The human myometrium and cervix contain a substantial amount of PGs. The main sulfated GAG species in the myometrium and cervix is DS, with fewer CS and HS $(32,33)$. The GAG concentration in the myometrium markedly decreases during pregnancy, however, the proportion of the myometrial PGs does not vary during the menstrual cycle and gestational periods $(34,35)$.

The predominant DSPG in the myometrium and cervix is decorin, which comprises two-thirds of the total myometrial PGs, although biglycan, versican and several HSPGs are also detectable (36). The concentration of decorin in the cervix markedly decreases during term pregnancy, despite total PG synthesis increasing in this period (37), indicating the involvement of decorin in cervical competency in early to mid gestation.

Syndecan-3 is localized not only to the plasma membrane of smooth muscle cells and myometrial vascular cells, but also to the ECM of the myometrium. $17 \beta$-Estradiol, but not progesterone, has been found to be able to stimulate the expression of the syndecan-3 gene transcript in the rat myometrium (37). In contrast to decorin, syndecan-3 expression in the myometrium is patchy and weak in the non-pregnant condition, but becomes stronger with the establishment of pregnancy and increases towards term, particularly during labor (38). These findings indicate that syndecan-3 is essential for active uterine contractions during parturition.

\section{Contribution of fibromodulin, versican and decorin to structural maintenance and growth of uterine fibroids}

Uterine fibroids (leiomyoma) are benign tumors that may affect human reproduction at various points, including implantation, placentation and fetal growth/positioning. Fibromodulin is a CS-bearing ECM PG with close homology to decorin and biglycan and contributes to the formation of collagen fibrillogenesis. During the proliferative phase of the menstrual cycle, fibromodulin is expressed in the uterine fibroid tissue at a two- to three-fold higher level than in the intact myometrium (39). Similarly, decorin, versican, syndecan-4 and ECM protein-2 are overexpressed in fibroid tissues compared with the intact myometrium (40). Decorin in the uterine fibroid is unique, as it contains longer GAG side chains and higher D-glucuronate/L-iduronate ratios compared with that in the intact myometrium $(41,42)$. Increase in the size of the uterine fibroids results in the weaker activity of the sulfated GAG-degrading enzymes, including neutral endoglycosidase and acid endoglycosidase (43). Notably, the administration of gonadotropin-releasing hormone analog, a therapeutic agent for uterine fibroids, was found to reduce fibromodulin expression in fibroid tissues to the levels in the intact myometrium (39), whereas all-trans retinoic acid, a promising agent for the treatment of fibroids, inhibited the proliferative activity 
of leiomyoma cells in a dose-dependent manner via the downregulation of versican expression (44). These findings indicate that certain PGs function as scaffolds for the growth and development of fibroid tissues.

Furthermore, endometrium with uterine fibroids exhibits poor decidualization. One possible explanation for this impaired endometrial decidualization is that TGF- $\beta 3$ secreted by uterine fibroids suppresses the thrombomodulin expression in endometrial stromal fibroblasts (9).

\section{Low myometrial syndecan-3 expression in dystocia}

As described in section 6, syndecan-3 is significant in the effective myometrial contractility during delivery (35). This view is supported by the finding that the expression of the myometrial syndecan-3 gene transcript and protein is lower in women who experienced prolonged labor than in those with active labor, while there were no significant differences in myometrial syndecan-3 expression between non-pregnant women and those with active labor (38).

\section{PGs in human placenta}

The major PGs in the human placenta are HSPGs (syndecans and perlecan) and DSPGs (decorin and biglycan) (45). Syndecan-1 is localized mainly to syncytiotrophoblasts of the placental villi (46). Decorin is expressed in the stromal compartment surrounding the fetal capillaries of chorionic villi, while biglycan is distributed in endothelial cells and smooth muscle cells of fetal capillaries (47). The expression level of syndecan-1 is upregulated in the process by which cytotrophoblasts differentiate into syncytiotrophoblasts (48).

Similar to early pregnancy, placental PGs are also critical in the maintenance of placental vascular integrity during late pregnancy, via the anticoagulant activities of HS and DS, which stimulate the anticoagulant factors anti-thrombin III and heparin cofactor II, respectively (49). Moreover, the results of in vitro experiments have shown that decorin inhibits the migration of human invading trophoblasts through insulinlike growth factor receptor I, while inhibiting the proliferation of these cells via receptors for epithelial growth factor or vascular endothelial growth factor (50), which are critical for hemochorial placental formation.

10. Amniotic fluid perlecan and agrin as potential biomarkers for premature rupture of fetal membranes

Perlecan (also known as HSPG2) is a large basement membrane PG with five structural domains homologous to several growth factors. Perlecan is able to modulate various molecules involved in lipid metabolism, cell adhesion and angiogenesis.

In addition to the amnion and chorion, a dense accumulation of perlecan is observed in the central area of Nitabuch's layer, where chorionic villi anchor the decidua basalis. The expression level of placental perlecan is highest in early gestation and decreases towards term pregnancy (51). Perlecan binds heparin/HS-interacting proteins on invading trophoblasts with high affinity and regulates their migration into the decidua (52).

It has been found that the C-terminal laminin-like globular domain of perlecan is cleaved to form bioactive fragments by matrix metalloproteinases with angiostatic activity on endothelial cells. Proteomic analysis has found that these bioactive perlecan fragments are detectable specifically in the amniotic fluid of pregnant women who experienced the premature rupture of fetal membranes, as well as another basement membrane HSPG, agrin (53). Premature rupture of fetal membranes is the pathological condition responsible for preterm deliveries and intrauterine infections, which cause neonatal morbidity and mortality. These basement membrane PGs are expected to be used as clinical biomarkers for the prediction of the premature rupture of fetal membranes.

\section{Preventive role of syndecan-1 against pre-eclampsia}

Pre-eclampsia is an obstetrical complication in which pregnant women develop hypertension with or without proteinuria. Pre-eclampsia causes serious maternal life-threatening conditions, including placental abruption, eclampsia and cerebral hemorrhage.

The expression level of HSPG syndecan-1 in syncytiotrophoblasts is reduced in patients with pre-eclampsia compared with those undergoing normal pregnancy (46). In support of this finding, mice lacking the syndecan-1 gene are fertile but exhibit fetal growth restriction which is frequently complicated with pre-eclampsia $(54,55)$. Mice lacking HS-3- $O$-sulfotransferase-1, the rate-limiting enzyme for anticoagulant HS synthesis, also exhibit fetal growth restriction without marked coagulopathy (56). These results indicate the contribution of HSPGs to placental integrity via anticoagulant and alternative pathways.

In humans, the expression level of decorin and CS/DS side chains in the term placenta is lower in women with pre-eclampsia compared with normal pregnancy $(47,57)$. By contrast, the plasma concentration of HSPG thrombomodulin is significantly higher in women with pre-eclampsia than in those with normal pregnancy, supporting the hypothesis that endothelial injury is one of the mechanisms underlying pre-eclampsia (58). Placental HSPGs and DSPGs may exert their functions for optimal vascular adaptation during gestation.

\section{Upregulation of perlecan in villi of gestational diabetes mellitus}

Placental villi in gestational diabetes mellitus express a higher level of perlecan and CS/DS than those in normal pregnancy (59). In contrast to the findings that hyperglycemia suppressed perlecan and HS synthesis in aortic and glomerular endothelial cells, high glucose concentration and osmotic pressure were found to enhance in vitro perlecan expression in cytotrophoblasts (59). The mechanism and significance for increased placental perlecan expression in gestational diabetes mellitus remain to be elucidated.

\section{Elevated amniotic fluid lumican concentration in fetus with Turner syndrome}

Lumican is highly expressed and its expression is elevated in certain types of cataract, one of the complications that occur in Turner syndrome. The lumican concentration has been shown to be higher in the amniotic fluid of Turner syndrome 
fetuses, although its correlation with postnatal cataract in this pathology is unknown (60).

\section{Conclusions}

There is a growing body of research which shows the essential roles of PGs in mammalian reproduction. The results of studies support the hypothesis that a deficiency in certain uterine PGs affects human fecundity. Several mice strains with deletion of the genes for certain PGs or GAG-biosynthetic enzymes undergo fetal mortality due to the lethal dysgenesis of the vital organs. The roles of these PGs in the human uterus remain largely unclear. Studies that use conditional knockout mice that specifically and transiently lose the endometrial gene expression of the PG core proteins or GAG-biosynthetic enzymes may provide more detailed information concerning the roles of individual PGs in reproduction.

\section{References}

1. Iozzo RV (ed): Proteoglycans: Structure, Biology and Molecular Interactions. Marcel Decker Inc., New York, 1990.

2. Aplin JD, Charlton AK and Ayad S: An immunohistochemical study of human endometrial extracellular matrix during the menstrual cycle and first trimester of pregnancy. Cell Tissue Res 253: 231-240, 1988.

3. Kelly FD, Tawia SA and Rogers PA: Immunohistochemical characterization of human endometrial microvascular basement membrane components during the normal menstrual cycle. Hum Reprod 10: 268-276, 1995.

4. Hoadley ME, Seif MW and Aplin JD: Menstrual-cycle-dependent expression of keratan sulphate in human endometrium. Biochem J 266: 757-763, 1990.

5. Kitaya K and Yasuo T: Dermatan sulfate proteoglycan biglycan as a potential selectin L/CD44 ligand involved in selective recruitment of peripheral blood CD16(-) natural killer cells into human endometrium. J Leukoc Biol 85: 391-400, 2009.

6. Nasciutti LE, Ferrari R, Berardo PT, et al: Distribution of chondroitin sulfate in human endometrium. Micron 37: 544-550, 2006.

7. Dassen H, Punyadeera C, Kamps R, et al: Progesterone regulation of implantation-related genes: new insights into the role of oestrogen. Cell Mol Life Sci 64: 1009-1032, 2007.

8. Yasuo T, Kitaya K, Yamaguchi T, et al: Possible role of hematopoietic CD44/chondroitin sulfate interaction in extravasation of peripheral blood CD16(-) natural killer cells into human endometrium. J Reprod Immunol 78: 1-10, 2008.

9. Sinclair DC, Mastroyannis A and Taylor HS: Leiomyoma simultaneously impair endometrial BMP-2-mediated decidualization and anticoagulant expression through secretion of TGF- $\beta 3$. J Clin Endocrinol Metab 96: 412-421, 2011.

10. Achache H, Tsafrir A, Prus D, et al: Defective endometrial prostaglandin synthesis identified in patients with repeated implantation failure undergoing in vitro fertilization. Fertil Steril 94: 1271-1278, 2010.

11. Yamaguchi T, Kitaya K, Daikoku N, et al: Potential selectin L ligands involved in selective recruitment of peripheral blood CD16(-) natural killer cells into human endometrium. Biol Reprod 74: 35-40, 2006.

12. Kitaya K, Yamaguchi T, Yasuo T, et al: Post-ovulatory rise of endometrial CD16(-) natural killer cells: in situ proliferation of residual cells or selective recruitment from circulating peripheral blood? J Reprod Immunol 76: 45-53, 2007.

13. Hanna J, Goldman-Wohl D, Hamani Y, et al: Decidual NK cells regulate key developmental processes at the human fetalmaternal interface. Nat Med 12: 1065-1074, 2006.

14. Isermann B, Hendrickson SB, Hutley K, et al: Tissue-restricted expression of thrombomodulin in the placenta rescues thrombomodulin-deficient mice from early lethality and reveals a secondary developmental block. Development 128: 827-838, 2001.

15. Drake BL and Loke YW: Isolation of endothelial cells from human first trimester decidua using immunomagnetic beads. Hum Reprod 6: 1156-1159, 1991.
16. Stortoni P, Cecati M, Giannubilo SR, et al: Placental thrombomodulin expression in recurrent miscarriage. Reprod Biol Endocrinol 8: 1,2010.

17. Ishiguro K, Kadomatsu K, Kojima T, et al: Syndecan-4 deficiency impairs the fetal vessels in the placental labyrinth. Dev Dyn 219: 539-544, 2000.

18. Germeyer A, Klinkert MS, Huppertz AG, et al: Expression of syndecans, cell-cell interaction regulating heparan sulfate proteoglycans, within the human endometrium and their regulation throughout the menstrual cycle. Fertil Steril 87: 657-663, 2007.

19. Inki P, Stenbäck F, Grenman S and Jalkanen M: Immunohistochemical localization of syndecan-1 in normal and pathological human uterine cervix. J Pathol 72: 1349-1355, 1994.

20. Bayer-Garner IB, Nickell JA and Korourian S: Routine syndecan-1 immunohistochemistry aids in the diagnosis of chronic endometritis. Arch Pathol Lab Med 28: 1000-1003, 2004

21. Lai TH, King JA, Shih IeM, et al: Immunological localization of syndecan-1 in human endometrium throughout the menstrual cycle. Fertil Steril 87: 121-126, 2007.

22. Kitaya K and Yasuo T: Immunohistochemistrical and clinicopathological characterization of chronic endometritis. Am J Reprod Immunol 66: 410-415, 2011.

23. Johnston-MacAnanny EB, Hartnett J, Engmann LL, et al: Chronic endometritis is a frequent finding in women with recurrent implantation failure after in vitro fertilization. Fertil Steril 93: 437-441, 2010.

24. Kitaya K and Yasuo T: Aberrant expression of selectin E, CXCL1, and CXCL13 in chronic endometritis. Mod Pathol 23: 1136-1146, 2010.

25. Kitaya K: Prevalence of chronic endometritis in recurrent miscarriages. Fertil Steril 95: 1156-1158, 2011.

26. Kaushal GP, Xiong X, Athota AB, et al: Syndecan-1 expression suppresses the level of myeloma matrix metalloproteinase-9. Br J Haematol 104: 365-373, 1999.

27. Saïdi H, Magri G, Nasreddine N, et al: R5- and X4-HIV-1 use differentially the endometrial epithelial cells HEC-1A to ensure their own spread: implication for mechanisms of sexual transmission. Virology 358: 55-68, 2007.

28. Berardo PT, Abrao MS, Souza ML, et al: Composition of sulfated glycosaminoglycans and immunodistribution of chondroitin sulfate in deeply infiltrating endometriosis affecting the rectosigmoid. Micron 40: 639-645, 2009.

29. Aghajanova L and Giudice LC: Molecular evidence for differences in endometrium in severe versus mild endometriosis. Reprod Sci 18: 229-251, 2011.

30. Vouk K, Smuc T, Guggenberger C, et al: Novel estrogen-related genes and potential biomarkers of ovarian endometriosis identified by differential expression analysis. J Steroid Biochem Mol Biol 125: 231-242, 2011.

31. Veillat V, Carli $\mathrm{C}$, Metz $\mathrm{CN}$, et al: Macrophage migration inhibitory factor elicits an angiogenic phenotype in human ectopic endometrial cells and triggers the production of major angiogenic factors via CD44, CD74, and MAPK signaling pathways. J Clin Endocrinol Metab 95: e403-e412, 2010.

32. Berto AG, Oba SM, Michelacci YM and Sampaio LO: Galactosaminoglycans from normal myometrium and leiomyoma. Braz J Med Biol Res 34: 633-637, 2001.

33. Westergren-Thorsson $\mathrm{G}$, Norman M, Björnsson S, et al: Differential expressions of mRNA for proteoglycans, collagens and transforming growth factor- $\beta$ in the human cervix during pregnancy and involution. Biochim Biophys Acta 1406: 203-213, 1998.

34. Hjelm A, Ekman-Ordeberg G, Barchan K and Malmström A: Identification of the major proteoglycans from human myometrium. Acta Obstet Gynecol Scand 80: 1084-1090, 2001.

35. Cabrol D, Breton M, Berrou E, et al: Variations in the distribution of glycosaminoglycans in the uterine cervix of the pregnant woman. Eur J Obstet Gynecol Reprod Biol 10: 281-287, 1980.

36. Uldeberg N, Malmström A, Ekman G, et al: Isolation and characterization of dermatan sulphate proteoglycan from human uterine cervix. Biochem J 209: 497-503, 1983.

37. Russo LA, Calabro SP, Filler TA, et al: In vivo regulation of syndecan-3 expression in the rat uterus by $17 \beta$-estradiol. J Biol Chem 276: 686-692, 2001.

38. Cluff AH, Byström B, Klimaviciute A, et al: Prolonged labor associated with lower expression of syndecan 3 and connexin 43 in human uterine tissue. Reprod Biol Endocrinol 4: 24, 2006. 
39. Levens E, Luo X, Ding L, et al: Fibromodulin is expressed in leiomyoma and myometrium and regulated by gonadotropinreleasing hormone analogue therapy and TGF- $\beta$ through Smad and MAPK-mediated signalling. Mol Hum Reprod 11: 489-494, 2005.

40. Pan Q, Luo X and Chegini N: Genomic and proteomic profiling I: leiomyomas in African Americans and Caucasians. Reprod Biol Endocrinol 5: 34, 2007.

41. Malik M and Catherino WH: Novel method to characterize primary cultures of leiomyoma and myometrium with the use of confirmatory biomarker gene arrays. Fertil Steril 87: 1166-1172, 2007.

42. Berto AG, Sampaio LO, Franco CR, et al: A comparative analysis of structure and spatial distribution of decorin in human leiomyoma and normal myometrium. Biochim Biophys Acta 1619: 98-112, 2003.

43. Wolańska M, Sobolewski K, Cechowska-Pasko M and Jaworski S: The activities of some glycosaminoglycan-degrading enzymes in uterine leiomyomas. Eur J Obstet Gynecol Reprod Biol 110: 73-78, 2003

44. Malik M, Webb J and Catherino WH: Retinoic acid treatmen of human leiomyoma cells transformed the cell phenotype to one strongly resembling myometrial cells. Clin Endocrinol 69 462-470, 2008

45. Achur RN, Valiyaveettil M, Alkhalil A, et al: Characterization of proteoglycans of human placenta and identification of unique chondroitin sulfate proteoglycans of the intervillous spaces that mediate the adherence of Plasmodium falciparum-infected erythrocytes to the placenta. J Biol Chem 275: 40344-40356, 2000 .

46. Jokimaa V, Inki P, Kujari H, et al: Expression of syndecan-1 in human placenta and decidua. Placenta 19: 157-163, 1998.

47. Swan BC, Murthi P, Rajaraman G, et al: Decorin expression is decreased in human idiopathic fetal growth restriction. Reprod Fertil Dev 22: 949-955, 2010.

48. Aronow BJ, Richardson BD and Handwerger S: Microarray analysis of trophoblast differentiation: gene expression reprogramming in key gene function categories. Physiol Genomics 6 : $105-116,2001$

49. Giri TK and Tollefsen DM: Placental dermatan sulfate: isolation, anticoagulant activity, and association with heparin cofactor II. Blood 107: 2753-2758, 2006.

50. Iacob D, Cai J, Tsonis M, et al: Decorin-mediated inhibition of proliferation and migration of the human trophoblast via different tyrosine kinase receptors. Endocrinology 149: 6187-6197, 2008.

51. Chen CP, Liu SH, Lee MY and Chen YY: Heparan sulfate proteoglycans in the basement membranes of the human placenta and decidua. Placenta 29: 309-316, 2008.

52. Rohde LH, Janatpore MJ, McMaster MT, et al: Complementary expression of HIP, a cell-surface heparan sulfate binding protein, and perlecan at the human fetal-maternal interface. Biol Reprod 58: 1075-1083, 1998.
53. Vuadens F, Benay C, Crettaz D, et al: Identification of biologic markers of the premature rupture of fetal membranes: proteomic approach. Proteomics 3: 1521-1525, 2003.

54. Stepp MA, Gibson HE, Gala PH, et al: Defects in keratinocyte activation during wound healing in the syndecan-1-deficient mouse. J Cell Sci 115: 4517-4531, 2002.

55. McDermott SP, Ranheim EA, Leatherberry VS, et al: Juvenile syndecan-1 null mice are protected from carcinogen-induced tumor development. Oncogene 26: 1407-1416, 2006.

56. Warda M, Zhang F, Radwan M, et al: Is human placenta proteoglycan remodeling involved in pre-eclampsia? Glycoconj J 25: 441-450, 2008

57. Stepanian A, Benchenni S, Beillat-Lucas T, et al: Search for an association between V249I and T280M CX3CR1 genetic polymorphisms, endothelial injury and preeclampsia: the ECLAXIR study. PLoS One 4: e6192, 2009.

58. Shworak NW, HajMohammadi S, de Agostini AI and Rosenberg RD: Mice deficient in heparan sulfate 3-O-sulfotransferase-1: normal hemostasis with unexpected perinatal phenotypes. Glycoconj J 19: 355-361, 2002.

59. Yang WC, Su TH, Yang YC, et al: Altered perlecan expression in placental development and gestational diabetes mellitus. Placenta 26: 780-788, 2005.

60. Mavrou A, Anagnostopoulos AK, Kolialexi A, et al: Proteomic analysis of amniotic fluid in pregnancies with Turner syndrome fetuses. J Proteome Res 7: 1862-1866, 2008.

61. Maeda D, Ota S, Takazawa Y, et al: Glypican-3 expression in clear cell adenocarcinoma of the ovary. Mod Pathol 22: 824-832, 2009.

62. Lysiak JJ, Hunt J, Pringle GA and Lala PK: Localization of transforming growth factor- $\beta$ and its natural inhibitor decorin in the human placenta and decidua throughout gestation. Placenta 16: 221-231, 1995.

63. Nishiu J, Tanaka T and Nakamura Y: Identification of a novel gene (ECM2) encoding a putative extracellular matrix protein expressed predominantly in adipose and female-specific tissues and its chromosomal localization to $9 \mathrm{q} 22.3$. Genomics 52: 378-381, 1998.

64. Fischer DC, Henning A, Winkler M, et al: Evidence for the presence of a large keratan sulphate proteoglycan in the human uterine cervix. Biochem J 320: 393-399, 1996.

65. Shankar R, Johnson MP, Williamson NA, et al: Molecular markers of preterm labor in the choriodecidua. Reprod Sci 17: 297-310, 2010.

66. Yasuo T, Yamaguchi T and Kitaya K: Progesterone induction of chondroitin sulfate proteoglycan aggrecan expression in human endometrial epithelial cells. J Steroid Biochem Mol Biol 122: 159-163, 2010

67. Yasuo T and Kitaya K: Effect of ovarian steroids on gene expression profile in human uterine microvascular endothelial cells. Fertil Steril 92: 709-721, 2009. 